\title{
Meteorological path planning using dynamic programming for a solar- powered UAV
}

\section{Conference Paper}

\section{Author(s):}

Wirth, Lukas; Oettershagen, Philipp; Ambuhl, Jacques; Siegwart, Roland

Publication date:

2015

Permanent link:

https://doi.org/10.3929/ethz-a-010584936

Rights / license:

In Copyright - Non-Commercial Use Permitted

Originally published in:

https://doi.org/10.1109/AERO.2015.7119284 


\title{
Meteorological Path Planning Using Dynamic Programming for a Solar-Powered UAV
}

\author{
Lukas Wirth $^{1}$, Philipp Oettershagen ${ }^{1}$, Jacques Ambühl ${ }^{2}$, Roland Siegwart ${ }^{1}$ \\ ${ }^{1}$ ETH Zurich \\ Autonomous Systems Lab \\ Leonhardstrasse 21 \\ 8092 Zurich \\ +41446327395 \\ philipp.oettershagen@mavt.ethz.ch \\ ${ }^{2}$ Federal Office of Meteorology and \\ Climatology MeteoSwiss \\ Operation Center 1, Postfach 257 \\ CH-8058 Zurich-Airport \\ +41792782540 \\ jacques.ambuehl@meteoswiss.ch
}

\begin{abstract}
Solar-powered Unmanned Aerial Vehicles (SUAV) designed for Low-Altitude Long-Endurance (LALE) applications provide potential multi-day continuous flight capability, but are generally prone to local meteorological impediments such as rain, strong winds or reduced solar irradiance. This paper therefore presents METPASS, the Meteorology-aware Trajectory Planning and Analysis Software for Solar-powered UAVs. METPASS optimizes largescale solar-powered UAV missions using a detailed consideration of meteorological effects: An optimal trajectory is found on a 3-D grid for given departure and arrival points by applying a Dynamic Programming approach and a cost function that considers environmental hazards, winds, solar radiation, aircraft parameters and flight time. The cost function is evaluated based on a kinematic and energetic UAV system model and forecast data from the European Centre for Medium-Range Weather Forecasts (ECMWF). The trajectoryplanning environment is applied to an envisioned fully autonomous and solar-powered crossing of the North Atlantic Ocean by AtlantikSolar, a 5.6m-wingspan SUAV developed at ETH Zurich. Results based on historical ECMWF weather data from 2012 and 2013 show that properly pre-optimized routes allow the Atlantic crossing even in case of significant global cloud coverage and that optimal routes can reduce the required flight time by up to $50 \%$ (from $106 \mathrm{~h}$ to $52 \mathrm{~h}$ ) by exploiting wind conditions.
\end{abstract}

\section{TABLE OF CONTENTS}

1. INTRODUCTION .1

2. METHODOLOGY AND IMPLEMENTATION..........2 3. FunCtional VALIDATION AND PARAMETER REFINEMENT .5

4. RESULTS. .7

5. CONCLUSION..........................................................9

REFERENCES..............................................................9

BIOGRAPHY . .10

\section{INTRODUCTION}

\section{Solar-powered Unmanned Aerial Vehicles}

Today's solar-powered Unmanned Aerial Vehicles (SUAV) combine highly-optimized airframes and solar-powered recharging systems to provide increased flight-endurance. They can be designed to provide multi-day continuous flight
- often termed perpetual-flight - as recently shown by the 3.2m-wingspan SkySailor [1] and Zephyr [2]. These demonstrations, together with ongoing technical improvements in solar cell, electrical propulsion and battery technology have focused attention on SUAVs and their application in Search and Rescue (SAR) missions, meteorological observations, wildfire detection and the creation of communication networks. High-Altitude LongEndurance (HALE) platforms functioning as low-cost atmospheric satellites have created growing interest among IT companies by providing a solution to the latter applications [3]. In contrast, Low-Altitude Long-Endurance (LALE) solar-powered UAVs are more suitable for SAR missions due to lower complexity, size and thus handlaunch capability. Within this scope, ETH Zurich is currently developing AtlantikSolar [4], a solar-powered LALE-UAV platform with the goal to provide multi-day continuous flight even under partially cloudy conditions and to demonstrate the performance by performing an Atlantic crossing in summer 2015. The aircraft and its specifications are presented in Figure 1 and Table 1.

However, a major challenge for SUAVs consists of their sensitivity to environmental conditions caused by their lightweight design, low airspeed and dependency on solar radiation. The capability to perform detailed mission pre-

Table 1. AtlantikSolar UAV specifications

\begin{tabular}{|l|l|}
\hline Wingspan & $5.65 \mathrm{~m}$ \\
\hline Weight & $7 \mathrm{~kg}$ \\
\hline Solar module area & $1.4 \mathrm{~m}^{2}$ \\
\hline Battery total energy & $700 \mathrm{Wh}$ \\
\hline Typical power consumption & $50 \mathrm{~W}$ \\
\hline Typical airspeed & $9 \mathrm{~m} / \mathrm{s}$ \\
\hline
\end{tabular}

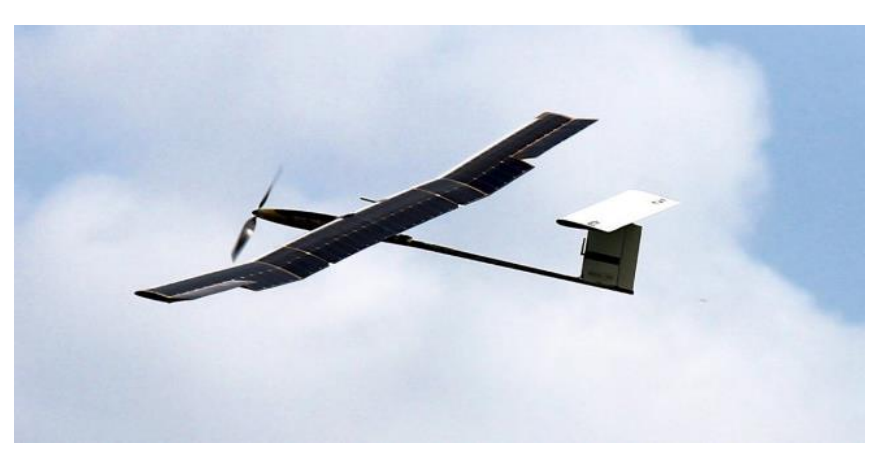




\section{Figure 1 - The AtlantikSolar UAV}

planning with respect to meteorological conditions is therefore indispensable for successful long-term operation of solar-powered UAVs.

\section{Contributions of this paper}

This paper contributes the Meteorology-aware Trajectory Planning and Analysis Software for Solar-powered UAVs (METPASS) developed at ETH Zurich to optimize largescale UAV missions with respect to flight safety and userdefinable performance-metrics (e.g. flight-time) by tightly integrating the UAV system model with meteorological considerations. METPASS integrates

1) A Dynamic Programming (DP) [15] optimization algorithm based on [5] that is extended to provide trajectory-optimization in altitude.

2) A cost function that defines trajectory-optimality by weighing safety (wind, rain, thunderstorms) and performance metrics.

3) A kinematic and energetic system model for solarpowered UAVs.

4) Methods to directly incorporate meteorological forecast data from the European Centre for Medium-Range Weather Forecasts (ECMWF).

By employing these components, METPASS allows to consider all aspects required for the safe operation of SUAVs. Feasibility analysis based on historical data, mission preplanning and in-flight path corrections can be performed. In contrast, previous literature addressed only individual aspects in mostly simplified conditions. In [6], minimization of flight time and fuel consumption in real wind conditions is addressed. As a fuel-powered UAV is considered, environmental impediments and solar radiation are not taken into account. [7] and [8] address the maximization of solar power generation in theoretical situations without any consideration of meteorological data. [9] and [10] investigate path optimization with respect to military threats or restricted areas.

\section{METHODOLOGY AND IMPLEMENTATION}

\section{Overview}

The path optimization problem solved by METPASS can be stated as follows: Given fixed departure and arrival coordinates, find a path and departure time which minimizes the total cost as defined by a cost function. The cost function includes environmental conditions such as solar radiation or precipitation as well as aircraft parameters like power consumption or State of Charge (SoC). Environmental conditions are estimated based on timevarying ECMWF forecast data in a three-dimensional grid. The system model includes flight kinematics with respect to horizontal wind, power generation through the solar modules and the power consumption of the aircraft. The required system components, namely the basic optimization algorithm, the cost function, the forecast data and the system

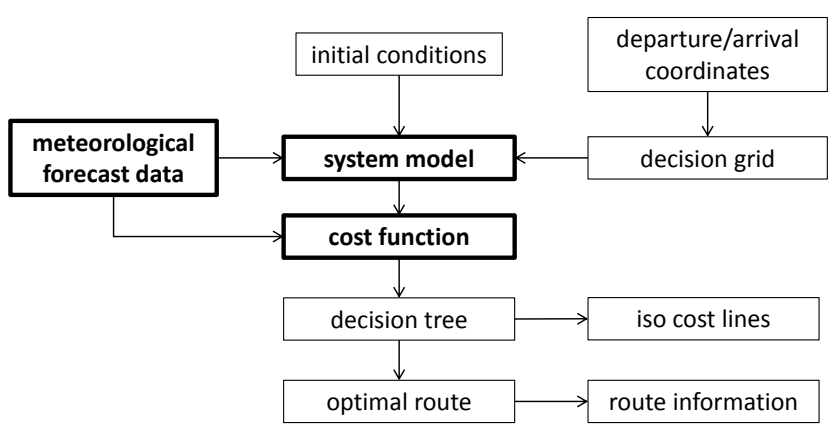

Figure 2 - Overview of the optimization software and the required components

model are explained in detail in the following sections. A graphical overview is given in Figure 2.

\section{Optimization Algorithm}

The optimization algorithm is an extended version of the Dynamic Programming based algorithm presented in [5] with the altitude as an additional optimization variable. The working principle can be shown on a basic example, where the goal is to find the shortest distance between Bell Island (CA) and Lisbon (PT) (Figure 3). In a first step, a threedimensional grid, connecting the departure and arrival points, is generated. The grid is horizontally divided into $\mathrm{i}$ slices of $\mathrm{j}$ vertices, and vertically into $\mathrm{k}$ levels. More information about scaling and resolution of the grid can be found in [14]. Starting from the departure node, the cost (in this example the travel distance) to each subsequent node is calculated and stored. Then, starting from the nodes in the third slice of the grid, the DP algorithm

$$
d_{i, j, k}=\min _{\mathrm{n} \in \text { slice }_{\mathrm{i}-1}}\left[\mathrm{~d}_{\mathrm{i}-1, \mathrm{n}}+\Delta_{\mathrm{i}-1, \mathrm{n}}^{\mathrm{i} \mathrm{j}, \mathrm{k}}\right]
$$

is applied to find the shortest total distance $d_{i, j, k}$ from the departure point to each node of the grid. This is done by minimizing the sum of the a priori known distances $d_{i-1, n}$ and the corresponding additional travel distance $\Delta_{i-1, n}^{\mathrm{i}, \mathrm{j}, \mathrm{k}}$. A decision tree consisting of globally optimal sub routes is thus built up which finally reaches the arrival point. The

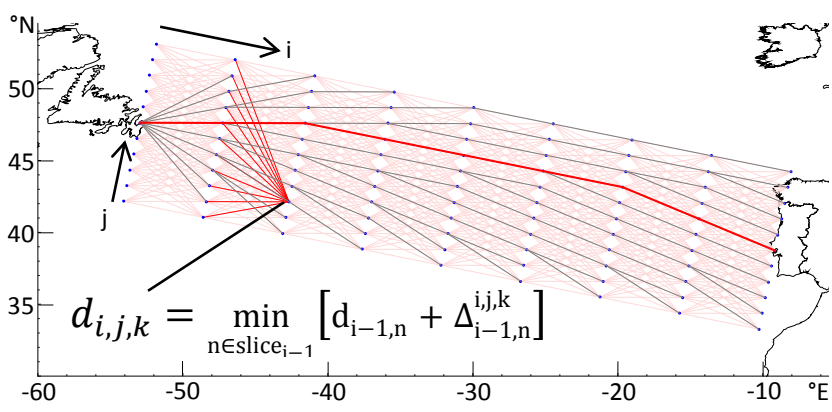

Figure 3 - Exemplary application of the optimization algorithm to a shortest path problem. The decision grid, 


\section{decision tree, a highlighted node and the resulting shortest path from Canada to Portugal are also shown.}

optimal path can then be extracted by going back up the tree from the arrival point.

In contrast to this simplified example, in which the cost is represented by the Euclidean distance that is calculated analytically as described in [5], the evaluation of the real cost function depends on high-resolution time-varying forecast data and an advanced system model. Thus the flight on each path segment needs to be simulated using numerical integration, which is a main expansion compared to [5], where the system model only consists of a polar diagram.

\section{Cost Function}

The cost function is a weighted combination of flight time, environmental costs and system costs. The environmental costs indicate an environmental threat to the airplane, which can be directly derived from the forecast data. This includes strong wind, wind gusts, humidity, precipitation and thunderstorms. The system costs are SoC, power consumption and power generation. To allow for a consistent summation and weighting, each of the costs $\dot{\mathrm{C}}_{\mathrm{k}}$ is expressed through

$$
\dot{\mathrm{C}}_{\mathrm{k}}=\mathrm{H}(\mathrm{x}) \cdot \frac{\exp \left(\frac{\mathrm{x}_{\mathrm{k}}-\alpha_{\mathrm{k}}}{\beta_{\mathrm{k}}-\alpha_{\mathrm{k}}} \varepsilon_{\mathrm{k}}\right)-1}{\exp \left(\varepsilon_{\mathrm{k}}\right)-1} .
$$

This normalizes every cost and allows to adjust its influence on the total cost using the parameters $\alpha_{\mathrm{k}}, \beta_{\mathrm{k}}$ and $\varepsilon_{\mathrm{k}}$. The parameters $\alpha_{\mathrm{k}}$ and $\beta_{\mathrm{k}}$ define the lower threshold and the upper limit, where the generated cost is bounded. By using the Heaviside function $\mathrm{H}(\mathrm{x})$, values $\mathrm{x}_{\mathrm{k}}$ below the threshold generate no cost, as they are not in a critical range and do not need to be considered. Values above the limit are considered as too dangerous for the aircraft and every violation will lead to a cancellation of the corresponding path. The exponent $\varepsilon_{\mathrm{k}}$ determines the shape of the cost curve as illustrated in Figure 4.

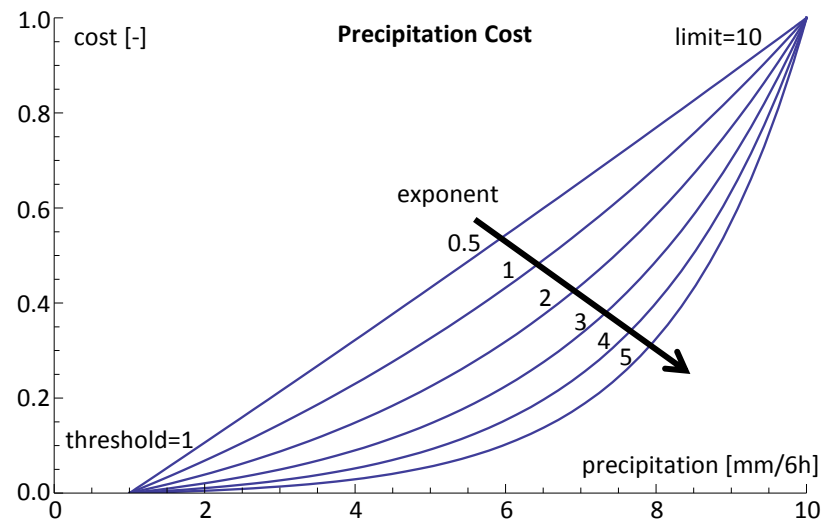

Figure 4 - Cost Function Example
The accumulated cost for a path segment is finally calculated by summing up all 9 costs to a total cost and integrating it over the flight time, as defined by

$$
C=\int_{t_{1}}^{t_{2}} \sum_{k=1}^{9} \dot{\mathrm{C}}_{\mathrm{k}} d t
$$

\section{Meteorological forecast data}

The meteorological data is obtained from ECMWF as historical data from 2012 to 2013. A horizontal resolution of $0.125^{\circ}$ with five altitude levels between 0 and $1600 \mathrm{~m}$ above sea-level and a time step of 6 hours are used. Additionally, forecast data with up to 5 days forecast range and a 3 hour time-step is available, which is updated every 12 hours. The data is linearly interpolated in all three spatial dimensions as well as time ${ }^{1}$. The used parameters are listed in Table 2. A more detailed description of how they are applied to the cost function and the system model can be found in [14].

\begin{tabular}{|c|c|c|c|}
\hline Parameter & Unit & Description & $\begin{array}{l}\text { Altitude } \\
\text { dependent }\end{array}$ \\
\hline Temperature & ${ }^{\circ} \mathrm{C}$ & & Yes \\
\hline Relative humidity & $\%$ & & Yes \\
\hline Zonal wind & $\mathrm{m} / \mathrm{s}$ & $\begin{array}{l}\text { Horizontal wind } \\
\text { from West to East }\end{array}$ & Yes \\
\hline Meridional wind & $\mathrm{m} / \mathrm{s}$ & $\begin{array}{l}\text { Horizontal wind } \\
\text { from South to North }\end{array}$ & Yes \\
\hline Wind gusts & $\mathrm{m} / \mathrm{s}$ & $\begin{array}{l}\text { Max. wind gust in } \\
\text { the last time step }\end{array}$ & No \\
\hline Total precipitation & $\mathrm{mm}$ & $\begin{array}{l}\text { Accumulated over } \\
\text { last time step }\end{array}$ & No \\
\hline $\begin{array}{l}\text { Convective available } \\
\text { potential energy }\end{array}$ & $\mathrm{J} / \mathrm{kg}$ & $\begin{array}{l}\text { Causing updrafts } \\
\text { and thunderstorms }\end{array}$ & No \\
\hline $\begin{array}{l}\text { Total solar radiation } \\
\text { (direct }+ \text { diffuse) }\end{array}$ & $\mathrm{J} / \mathrm{m}^{2}$ & $\begin{array}{l}\text { Accumulated over } \\
\text { last time step }\end{array}$ & No \\
\hline $\begin{array}{l}\text { Direct solar } \\
\text { radiation }\end{array}$ & $\mathrm{J} / \mathrm{m}^{2}$ & $\begin{array}{l}\text { Accumulated over } \\
\text { last time step }\end{array}$ & No \\
\hline
\end{tabular}

Table 2. Forecast Parameters received from ECMWF

\section{System Model}

An overview of the system model is given in Figure 5. The main components are the power balance estimation, including the evaluation of solar power generation, system power consumption and SoC, and the flight kinematics with respect to wind speed and airspeed. The flight planner represents the decision logic of the UAV, which determines the airspeed of the aircraft depending on the system state. The airspeed may be increased in presence of strong headwind in order to maintain a certain ground speed or if there is excessive solar power available, the battery is already fully charged and the aircraft is not allowed to gain additional altitude. For the calculation of the power generation, direct and diffuse solar radiation are considered separately. The incident angle of the direct radiation is

\footnotetext{
${ }^{1}$ The solar radiation is an exception as it changes much faster than all other meteorological parameters, which cannot be sufficiently tracked by a 3hour time step. The model-based estimation of current radiation values is described in [14].
} 
calculated for every solar module using the solar radiation models presented in [11], [12] and [14] under consideration of the aircraft geometry. The radiation onto the surface is then calculated using the cosine of the incidence angle. For the diffuse part of the radiation, the surface is assumed to be horizontal and thus the incidence angle is neglected. With

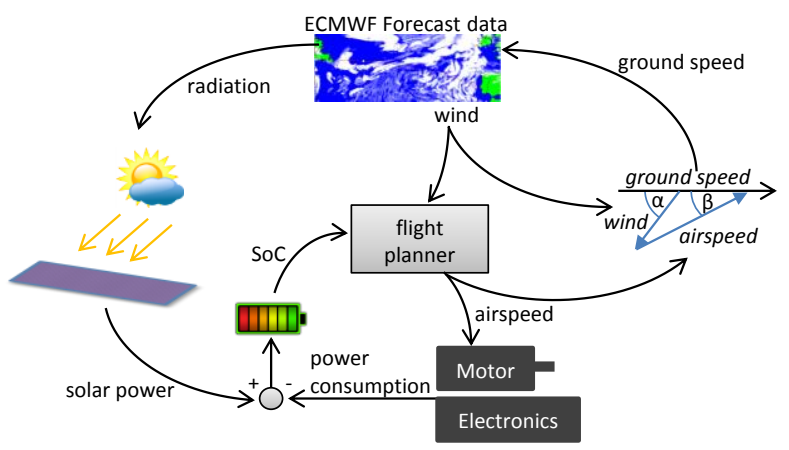

Figure 5 - Overview of the system model

the total area $A$ of the solar modules and the efficiencies of the solar cells $\eta_{\text {cell }}$ and the Maximum Power Point Trackers $\eta_{\text {MPPT }}$, the total incoming power is

$$
\mathrm{P}_{\text {solar }}=\left(\mathrm{I}_{\text {diff }}+\mathrm{I}_{\text {direct }} \cdot \cos \varphi\right) \cdot \mathrm{A} \cdot \eta_{\text {cell }} \cdot \eta_{\mathrm{MPPT}} \cdot
$$

The overall level-flight power consumption of the UAV depends on airspeed $v$ and altitude and thus air density $\rho$. It is generally given by

$$
P_{\text {level }}(\rho, v)=\frac{P_{\text {prop }}(\rho, v)}{\eta_{\text {prop }}(\rho, v)}+P_{a v}+P_{\text {pld }},
$$

where $P_{\text {prop }} / \eta_{\text {prop }}$ determines the required electrical propulsion power, and $P_{a v}$ and $P_{p l d}$ are avionics and payload power respectively. In our case, the dependence of
$P_{\text {level }}$ on the airspeed $v$ is modelled through

$$
\mathrm{P}_{\text {level }}\left(\rho_{0}, v\right)=\mathrm{C}_{2} \cdot \mathrm{v}^{2}+\mathrm{C}_{1} \cdot \mathrm{v}+\mathrm{C}_{0},
$$

which is identified directly from AtlantikSolar power measurement test flights performed at constant altitude and thus air density $\rho_{0}$. The scaling to different altitudes or air densities is done according to [13] using

$$
P_{\text {level }}(\rho, v)=\sqrt{\frac{\rho_{0}}{\rho}} \cdot\left[C_{2} \cdot v^{2} \cdot \frac{\rho}{\rho_{0}}+C_{P_{1}} \cdot v \cdot \sqrt{\frac{\rho}{\rho_{0}}}+C_{0}\right]
$$

With regard to the climb rate $\dot{h}$, the total flight power $P_{\text {flight }}$ is given by

$$
P_{\text {flight }}(\rho, v)=P_{\text {level }}(\rho, v)+\frac{m \cdot g \cdot \dot{h}}{\eta_{\text {climb }}},
$$

with the airplane mass $m$ and the climbing efficiency $\eta_{\text {climb }}$. The SoC is updated based on the power balance given by

$$
\operatorname{So} C=\frac{P_{\text {solar }}-P_{\text {flight }}}{E_{\text {battery }}} \cdot \eta_{\text {charge }}
$$

with the total energy of the battery $E_{\text {battery }}$ and the battery efficiency $\eta_{\text {charge }}$.

The flight kinematics include calculation of the wind correction angle and the resulting ground speed. More information about the system model may be found in [14].

\section{Implementation}

The implementation of the METPASS software, including data import and a versatile GUI, is done in Mathematica. The main optimization algorithm runs as compiled $\mathrm{C}$ code for the purpose of speed with full multicore support. Calculation times between 10 and 20 minutes are achieved for the cases presented in this paper with a quad-core Intel
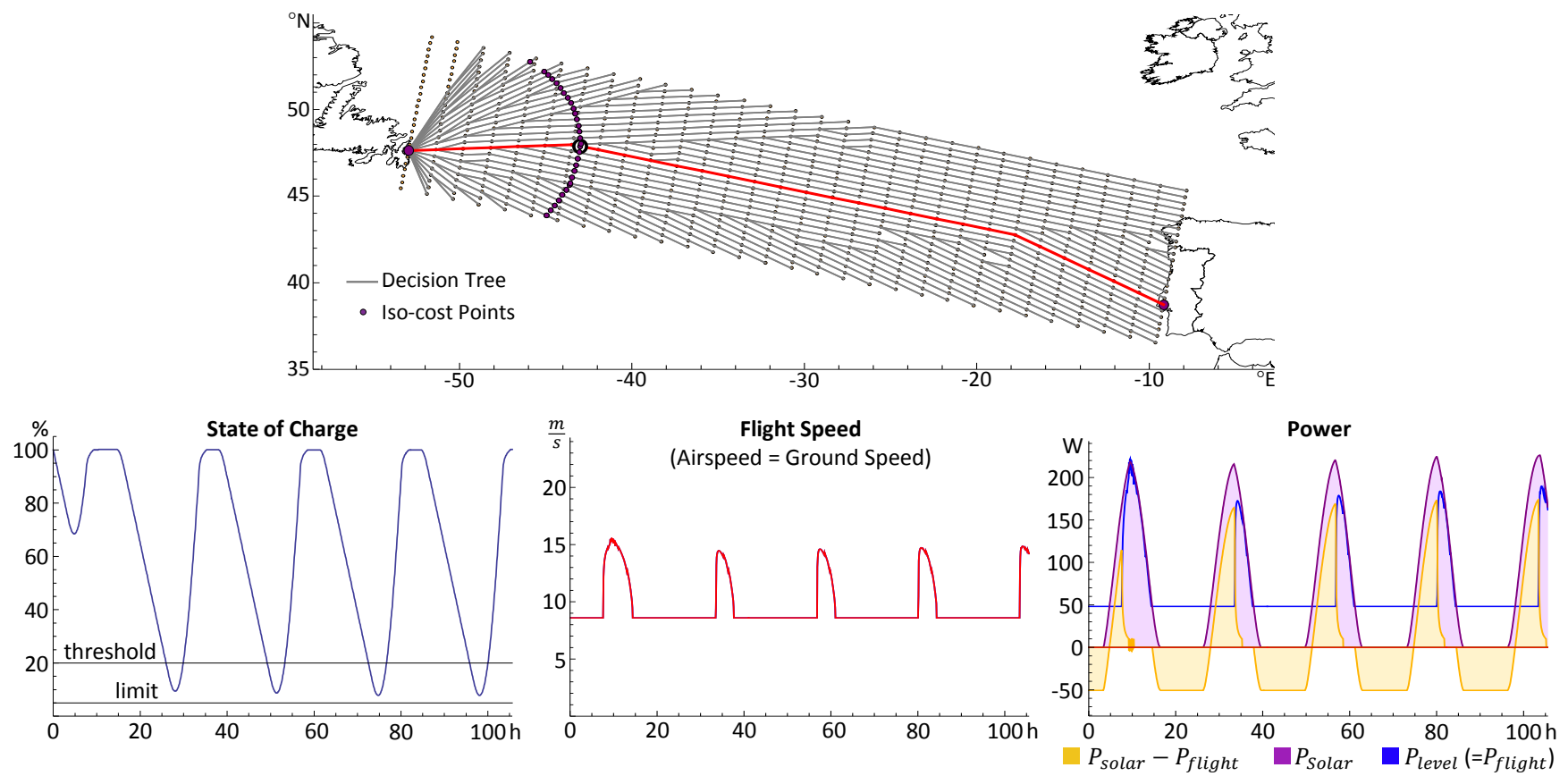

Figure 6 - Results of the first submodule test (2D, no altitude changes): Only the time cost is activated and all meteorological parameters are set to default values. The decision tree and the shortest path are calculated. The purple iso-cost points build a circle around the departure point. SoC and power consumption fluctuate periodically and the airspeed is increased if there is excessive solar power available and the battery fully charged. 
Xeon processor and 8GB memory. Meteorological data is automatically imported from text files and the resulting optimal path can be exported into a text file for direct dispatch to the UAV via a standard ground control station.

\section{Functional VAlidation ANd PARAMETER REFINEMENT}

\section{Procedure}

Following the software implementation, the functionality of the system is validated and a refinement of the cost parameters can be performed. This includes the following steps:

1) To independently verify the functionality of the system model and the optimization with respect to individual cost function components, submodules that activate only a subset of the cost components and corresponding environmental parameters are set-up.

2) Submodules are combined to validate the interaction, which is an essential requirement for the altitude decision-making.

3) With all submodules running, the full cost function and all environmental parameters can be employed. In order to balance the sensitivity of the cost function, three historical cases are chosen as training data for the refinement of the cost function parameters.

\section{Submodule Tests}

In the first submodule test, the required flight time is the only active cost. All forecast parameters are set to default values. It can be observed that the fastest path is found successfully and the system model produces the expected results (Figure 6). In particular, the output of the flight planner can be observed, which increases the airspeed as soon as the battery is fully charged and there is excessive solar power available. The total flight time under these nowind conditions is about 106 hours, the total distance is $3650 \mathrm{~km}$.

In a second submodule test, the flight time is still the only active cost, but the wind conditions from the ECMWF forecast data are considered. Thus the flight kinematics part of the system model and the three-dimensional path optimization under time-varying wind conditions can be observed. Figure 7 shows the optimal path with departure on May 30, 2013 10.00 AM UTC including the optimal flight altitude. The ground speed is usually maintained much higher than the airspeed, which shows that the wind conditions are exploited to minimize the flight time resulting in a total flight time of less than 60 hours.
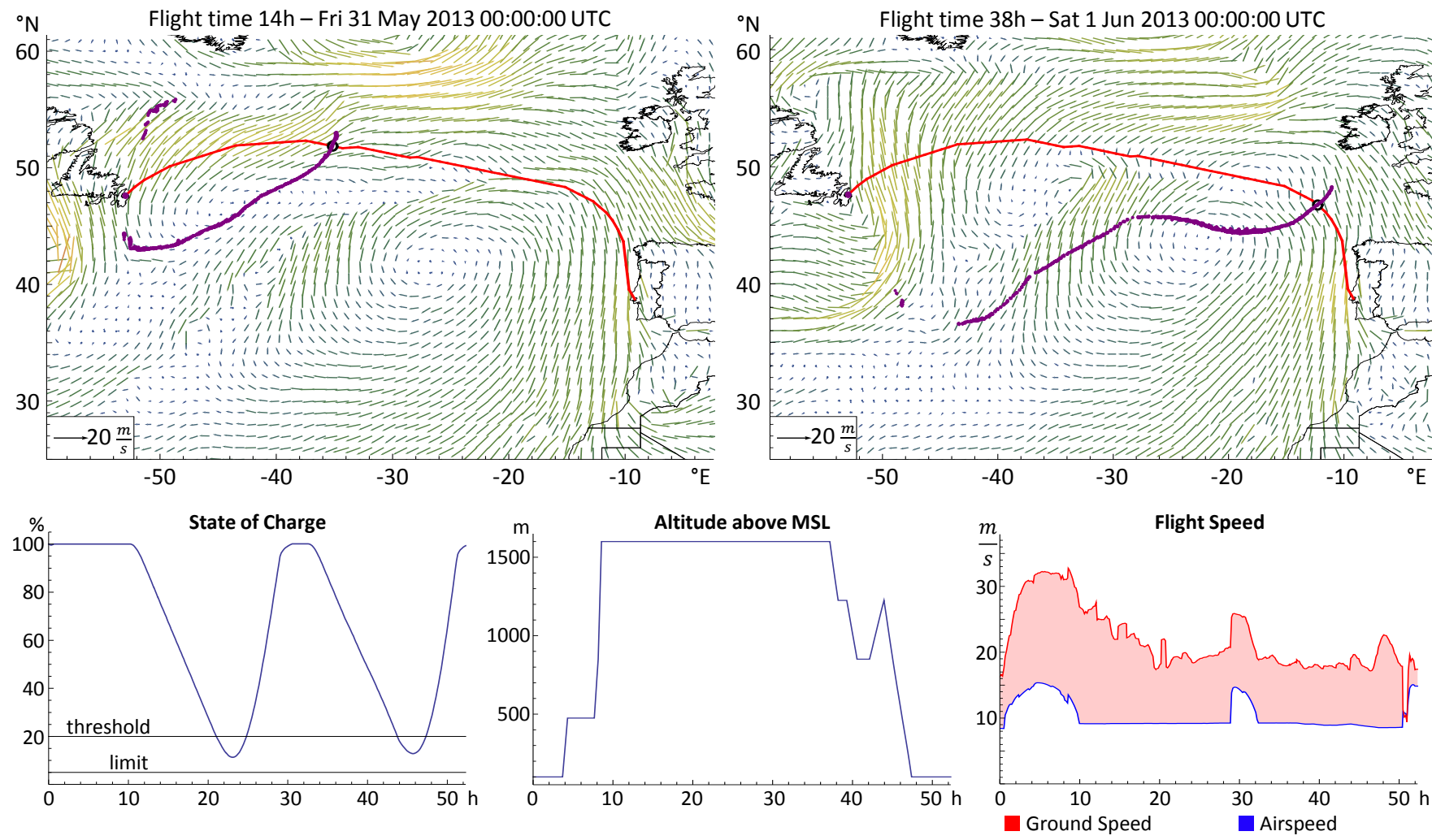

Figure 7 - Results of the second submodule test: Only the time cost is activated, but the wind conditions are taken into account. The resulting path minimizes the flight time by exploiting the wind conditions, including altitude changes. Ground speed is typically significantly higher than airspeed. The dependency of the iso-cost points on the wind conditions is clearly visible. 

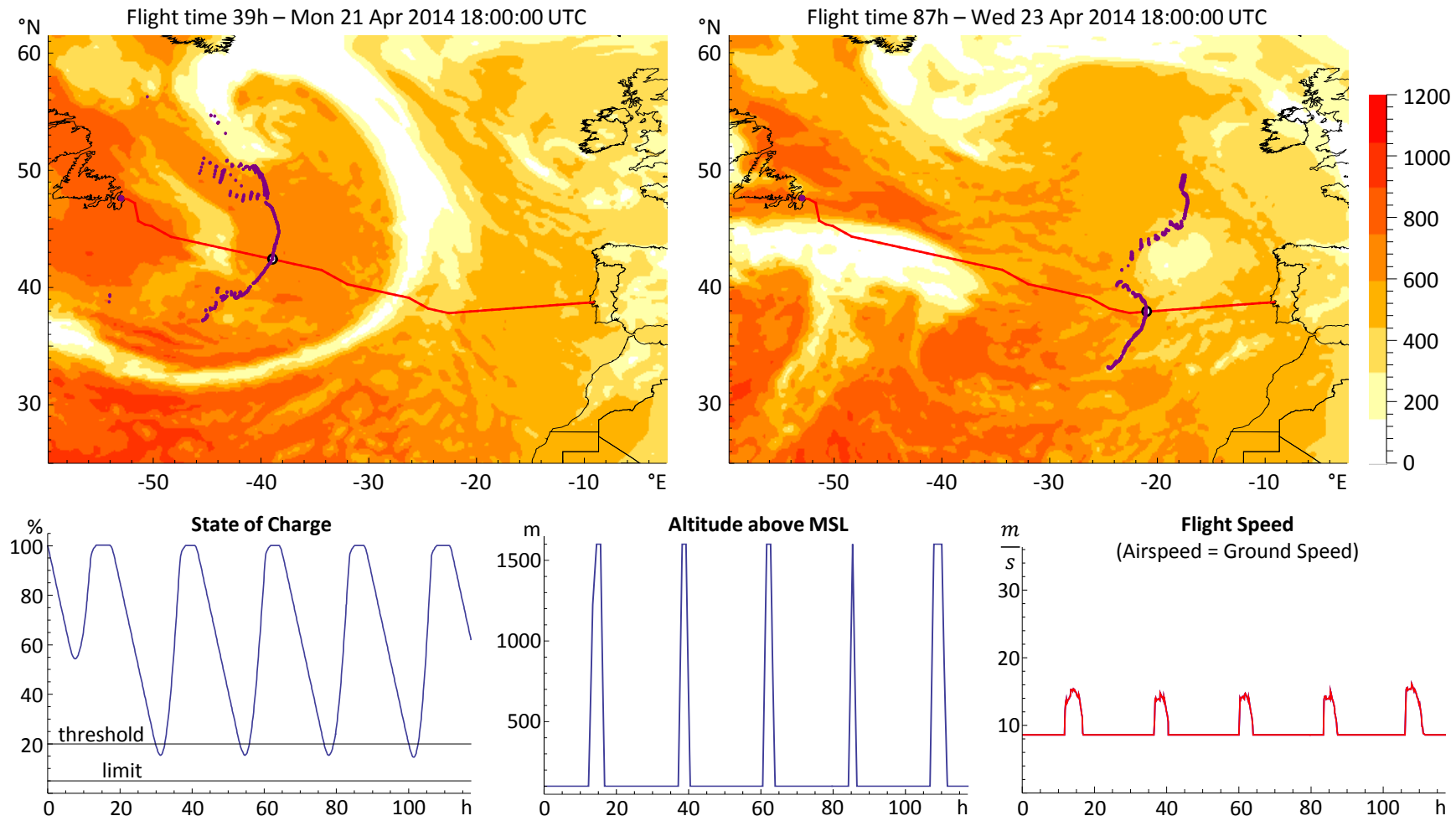

Figure 8 - Combination of submodules: Considered costs are solar radiation, power consumption and flight time. Only the weather parameters direct and total radiation are taken into account. The altitude changes depending on the SoC. The iso-cost points illustrate the influence of the radiation on the total cost.
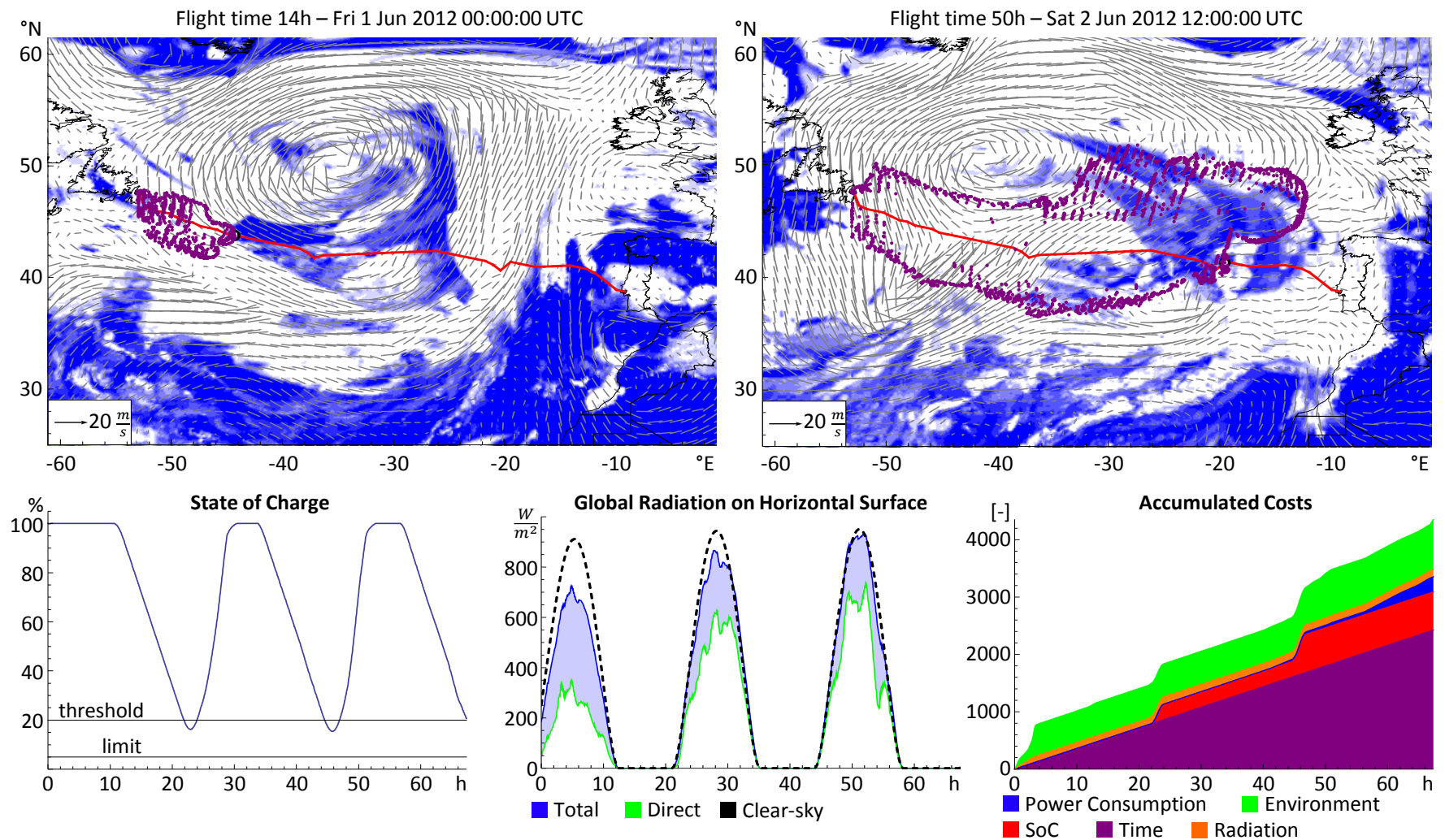

Figure 9 - Result of the historical case used for parameter refinement: The chosen path exploits wind conditions while providing enough solar radiation to fully charge the battery. The accumulated cost plot shows which cost parameters exceed the threshold. Only the time cost is constantly increasing. 
A combination of submodules employing several costs, namely solar radiation, power consumption and flight time, is illustrated in Figure 8. The applied meteorological parameters are only direct and total solar radiation. It can be observed that the optimal path follows highly radiated areas during the day and the shortest distance at night. The altitude changes show that the interaction between the costs for power consumption and flight time works as desired. At night, the lowest altitude level is chosen in order to minimize the power consumption. During the day, as soon as the battery is fully charged and there is excessive solar power available, the top altitude level is chosen, which has the advantage of increased airspeed at a given power consumption as well as storage of potential energy. A more detailed description of the different submodule tests may be found in [14].

\section{Parameter Refinement}

The parameter refinement starts with estimated initial values, which were either recommended by meteorologists or by the aircraft operators. The most important adjustments concern humidity, precipitation and convective available potential energy (CAPE). Both humidity and precipitation are higher than originally expected and thus dominate the optimization, which is why the corresponding sensitivity had to be reduced. In contrast, the CAPE values never even reached the threshold, wherefore the sensitivity could be increased. One example of a historical case, which was used for the refinement of the cost function parameters, is shown in Figure 9. Two more examples can be found in [14]. As these cases were used for the cost function refinement, the outcome of the path optimization is certainly as expected. In general, the wind speed is so high compared to the airspeed of the aircraft, that the wind conditions inevitably have the strongest impact on the path optimization. Nevertheless, the path follows regions with low cloud coverage at day, such that the battery is reliably charged. As the accumulated cost shows, most parameters can usually be held below the cost threshold.

\section{RESULTS}

\section{Historical Weather Data: Optimal and Marginal Conditions}

METPASS was applied to historical ECMWF weather data from the years 2012 and 2013 in order to identify the full range of conditions under which a crossing of the North Atlantic using the AtlantikSolar UAV is feasible. Identifying optimal and marginal border-cases together with their respective performance metrics minimum SoC, total accumulated cost and required flight time supplies key decision criteria when evaluating the conditions before an Atlantic crossing. Figure 11 shows an identified exemplary optimal border case on July 13th, 2012: The chosen route closely follows the orthodrome, with tailwind reducing the flight time by more than $50 \%$ to 52 hours. The accumulated cost is $C=2200$ and mainly consists of the time cost, which indicates that all other cost parameters usually stay below the safety threshold. This is confirmed by the minimum State of Charge, which always stays above
$17.2 \%$. In contrast, the exemplary June 4, 2013 test case exhibits marginal conditions (Figure 13, see Appendix). Here, the resulting path deviates significantly from the orthodrome, resulting in a flight time of $86 \mathrm{~h}$. This is due to severe cross and headwind, as well as high humidity and low solar radiation and therefore a low minimum State of Charge of only $7.1 \%$. Although it is guaranteed that none of the cost parameters ever reaches the defined critical limit, the total accumulated cost amounts to $C=17800$. The analysis of further border-cases yields the average optimal and marginal performance metrics of Table 3, which can be used to rank METPASS trajectory performance and decide upon the feasibility of launching AtlantikSolar on a certain date. This table is of course only valid for the specific cost function and UAV performance parameters presented in this paper.

\section{Table 3. Key trajectory performance metrics delivered by METPASS for typical optimal and marginal flight conditions}

\begin{tabular}{|l|l|l|}
\hline Test Case & Optimal & Marginal \\
\hline Expected minimum SoC & $>17 \%$ & $<8 \%$ \\
\hline Expected total cost & $<3000$ & $>15000$ \\
\hline Expected flight time & $<60 \mathrm{~h}$ & $>80 \mathrm{~h}$ \\
\hline
\end{tabular}

\section{Historical Weather Data: Seasonal Dependency of Results}

To assess the seasonal dependency of the performance metrics for an Atlantic crossing, METPASS trajectory optimizations were run in $6 \mathrm{~h}$ steps for the whole range from May $31^{\text {st }}$ to August $8^{\text {th }}$ for which historical ECMWF data was available. Figure 10 shows the minimum battery State of Charge for each date. It can be inferred that the current aircraft performance from Table 1 and Section 2 provides sufficient feasible launch dates for an Atlantic crossing in a window from mid-May to end-July when requiring a minimum State of Charge margin of $10 \%$. Additional analysis of the optimized routes yields a minimum and average flight time of $52 \mathrm{~h}$ and $78 \mathrm{~h}$ respectively versus the $106 \mathrm{~h}$ for the no-wind sub-test of Section 3.

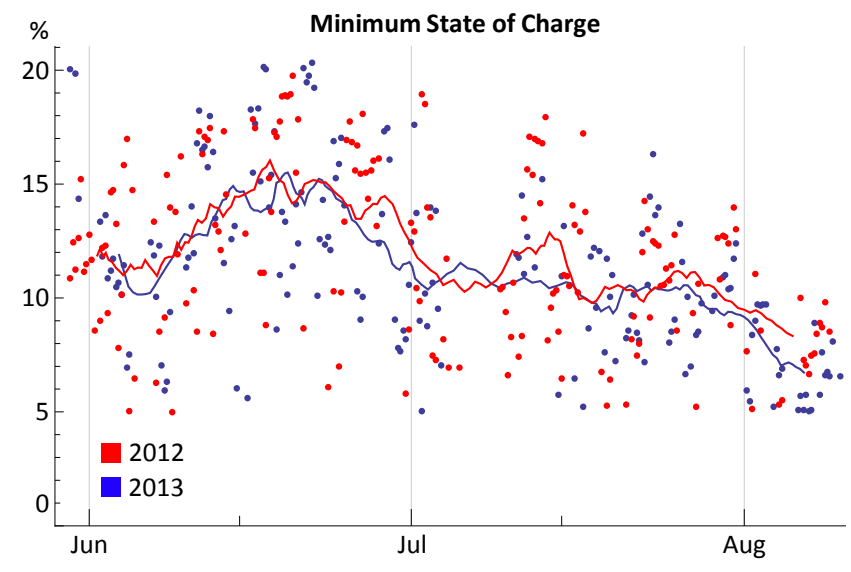

Figure 10 - Minimum battery State of Charge (SoC) versus date, for the whole range where meteorological data was available for the years 2012-2013. The solid line represents averaged SoC. 

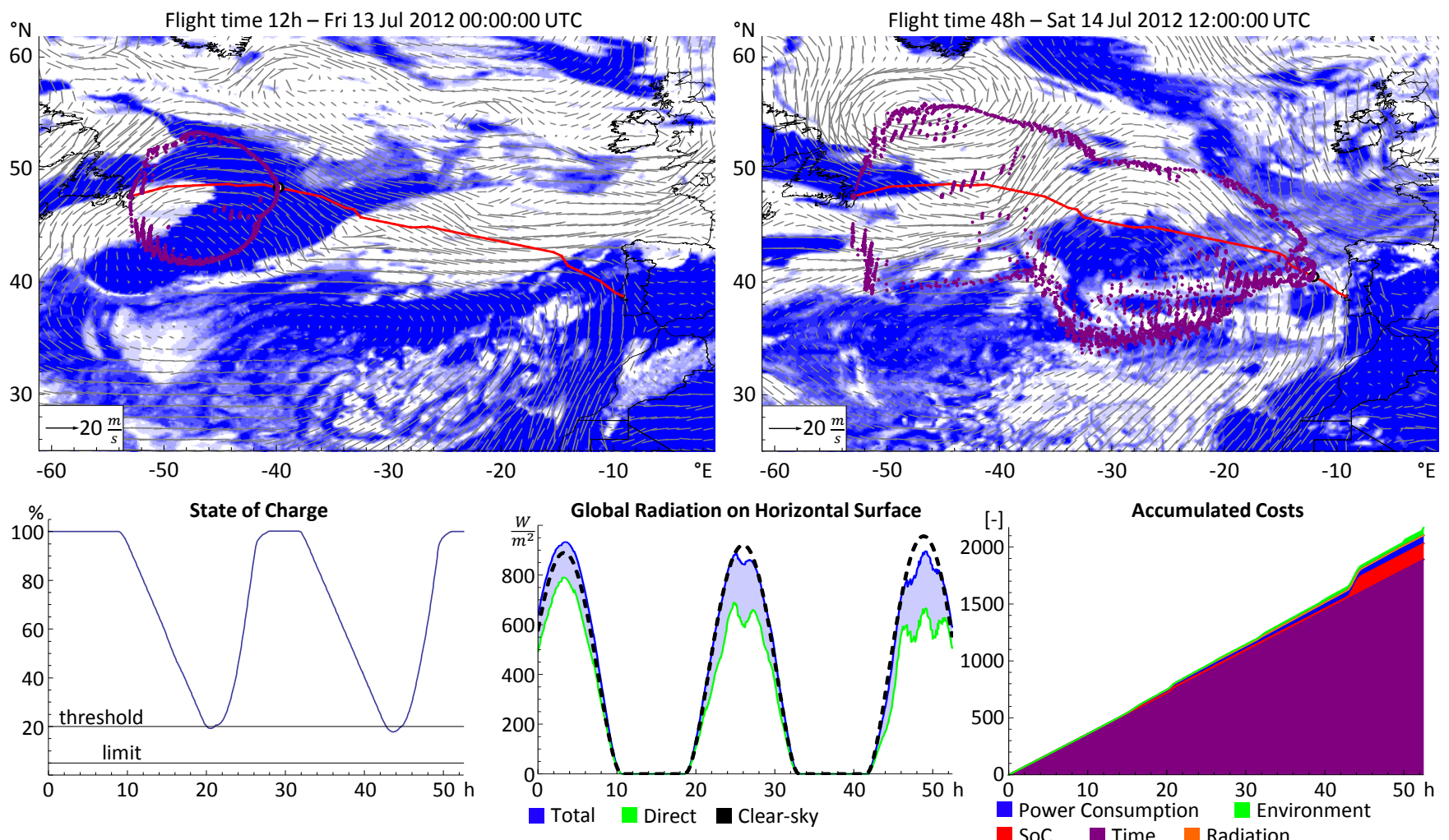

Figure 11 - The results for the case with optimal weather conditions show a path close to the orthodrome with a flight time of only 52 hours. The SoC barely enters the critical zone due to the high solar radiation. The accumulated cost consists mainly of the time cost, which indicates that all other cost parameters mostly stay below the safety-threshold.

Forecast Data: Real-Time Route Re-Planning

In addition to long-term pre-planning, METPASS can be used to perform UAV launch-time optimization as well as in-flight route-correction. In contrast to the previous examples, real forecast data is now used, which means that the forecast data is updated every 24 hours or more depending on the required accuracy. For real missions, the optimal launch time is as important as the path itself. By running the optimization algorithm for different departure times, the minimum total cost can be found. Figure 12 shows a plot of the total cost for a 50 hour time window around April 21, 2014, where the minimum is chosen for starting the simulation and performing a potential UAVlaunch. After 9 hours, new forecast data is available and the path optimization can be started with the current simulation states as initial values. This path correction, also illustrated in Figure 12, is repeated every 24 hours until the destination is reached successfully.
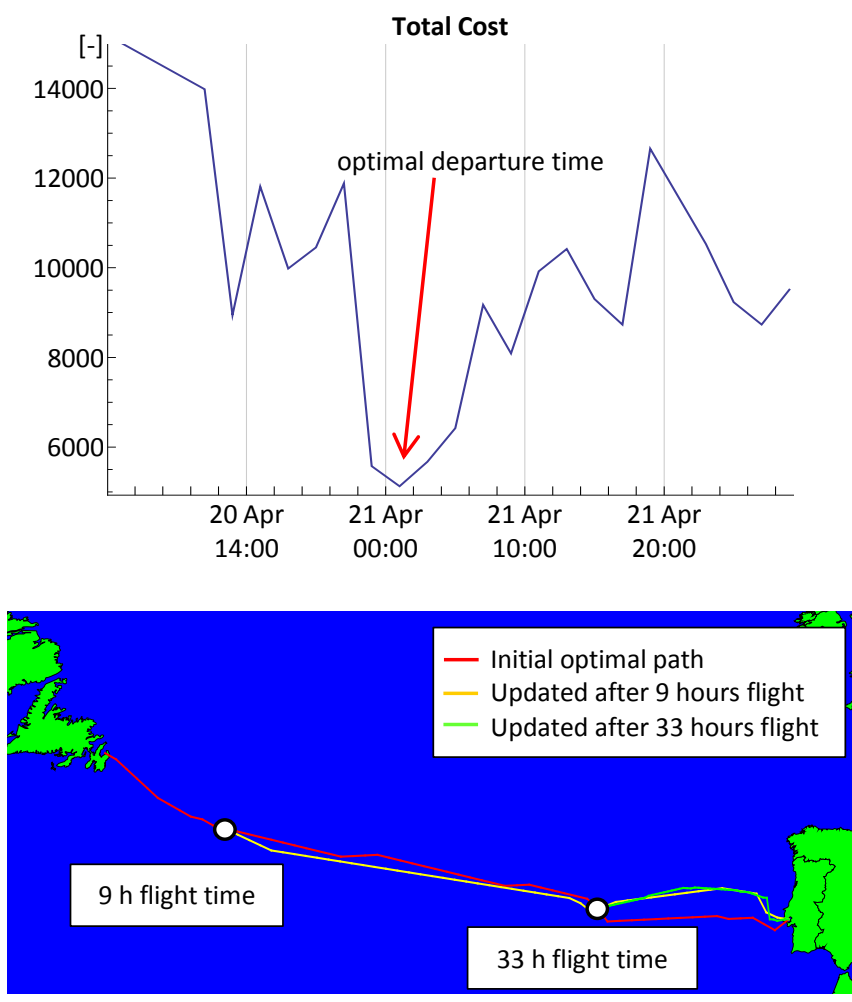

Figure 12 - Real-time example with forecast data on April 21, 2014 with start time optimization and in-flight path updates. 


\section{Conclusion}

The Meteorology-aware Trajectory Planning and Analysis Software for Solar-powered UAVs (METPASS) presented in this paper optimizes large-scale UAV missions with respect to a comprehensive cost function that, first, optimizes routes with respect to user-definable performance metrics such as flight-time and, second, increases flight safety with respect to meteorological impediments by incorporating precise historical or forecast ECMWF weather data. METPASS is applied to optimize a $4000 \mathrm{~km}$ route for the AtlantikSolar UAV's envisioned crossing of the North Atlantic Ocean. Results yield feasible routes from ca. mid-May to end-July, and properly planned routes lead to a wind-induced reduction in flight time of $50 \%$ while clearly avoiding meteorological impediments. In addition, METPASS is a vital instrument for providing key short-term decision criteria by taking into account the current weather forecasts for the entire North Atlantic: Through continuous pre-flight analysis of expected total trajectory cost and flight time, METPASS will be used to determine the exact optimum launch date for the AtlantikSolar UAVs, and will also perform regular re-optimizations of the route that can be directly dispatched to the airborne UAVs via a satellite communication link. The planning methodology itself is neither limited to large-scale missions nor to solar-powered UAVs, and could thus for example be extended towards aircraft trajectory planning in meteorologically-challenging smaller-scale environments such as alpine areas.

\section{ACKNOWLEDGEMENTS}

This work was supported by the EU-FP7 research projects ICARUS and SHERPA as well as a number of project partners and generous individuals, see [4].

\section{REFERENCES}

[1] A. Noth, "Design of solar powered airplanes for continuous flight", PhD thesis, ETH Zurich, 2008.

[2] QinetiQ, QinetiQ files for three world records for its Zephyr Solar powered UAV, QinetiQ Press Release, 2010. [Online].

[http://www.qinetiq.com/media/news/releases/Pages/three -world-records.aspx]

[3] E. Ackerman, Giant Solar-Powered UAVs are Atmospheric Satellites, IEEE Spectrum, 2013. [Online]. [http://spectrum.ieee.org/automaton/robotics/aerialrobots/giant-solar-powered-uavs-are-atmosphericsatellites]

[4] AtlantikSolar, ETH Zurich, 2014. [Online]. [http://www.atlantiksolar.ethz.ch/]
[5] J. Ambühl, "Sailing the virtual Bol d'Or", Scientific Report MeteoSwiss, 98, 44 p. 2014. [Online].

[http://www.meteoswiss.admin.ch/web/de/forschung/publ ikationen/meteoschweiz_publikationen/scientific_reports. html]

[6] J. C. Rubio, S. Kragelund, "The trans-pacific crossing: Long range adaptive path planning for UAVs through variable wind fields", Digital Avionics Systems Conference, 2003.

[7] S. Hosseini, R. Dai, M. Mesbahi, "Optimal path planning and power allocation for a long endurance solar-powered UAV", American Control Conference, 2013.

[8] A. T. Klesh, P. T. Kabamba, "Solar-powered aircraft: Energy-optimal path planning and perpetual endurance", Journal of Guidance, Control, and Dynamics, 2009.

[9] S. A. Bortoff, "Path planning for UAVs", American Control Conference, 2000.

[10] Yao-Hong Qu, Quan Pan, Jian-guo Yan, "Flight path planning of UAV based on heuristically search and genetic algorithms", Industrial Electronics Society, 2005.

[11] C. Cornwall, Solar Equations Document, Earth System Research Laboratory, 2014. [Online]. [http://www.esrl.noaa.gov/gmd/grad/solcalc/solareqns.PD $\mathrm{F}]$

[12] H. S. Fischer, H. Gilgen, "DACHRad - Berechnung der direkten Sonneneinstrahlung in Deutschland, Österreich und der Schweiz", Bulletin of the Geobotanical Institute ETH, 2002.

[13] R. Grundmann, "Flugmechanik" lecture notes, Technische Universität Dresden, 2014. [Online]. [http://tu-dresden.de/die_tu_dresden/fakultaeten/fakultaet _maschinenwesen/ilr/tfd/studium/dateien/Flugmechanik_ V.pdf]

[14] L. Wirth, "Meteorological path planning using Dynamic Programming for a solar-powered Atlantic-crossing unmanned aerial vehicle" M.Sc. thesis, ETH Zurich, 2014.

[15] R. Bellman, "On a routing problem" RAND CORP SANTA MONICA CA, 1956. 


\section{BIOGRAPHY}

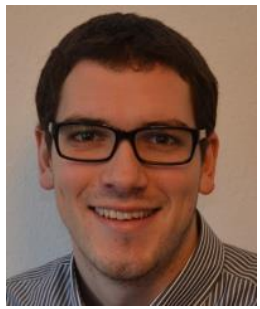

Lukas Wirth received a M.Sc. in Mechanical Engineering from ETH Zurich in 2014 with emphasis on Robotics, Systems and Control. His master thesis about meteorological path planning is the foundation of this paper. He is working for a company involved in the development of Personal Robots.

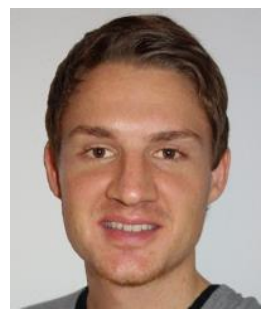

Philipp Oettershagen received a M.Sc. in Aerospace Engineering from California Institute of Technology in 2010 and a Diploma (Dipl.-Ing.) in Aerospace Engineering from University of Stuttgart, Germany, in 2011. He is currently a research assistant and PhD-student at ETH Zurich's Autonomous Systems Lab, where he is leading the AtlantikSolar Unmanned Aerial Vehicle (UAV) project. Besides the conceptual design and system engineering of high-performance solar-powered $U A V s$, he is interested in using in-flight estimations of the often highly cluttered and dynamic meteorological environment for trajectory planning and UAV decision making.

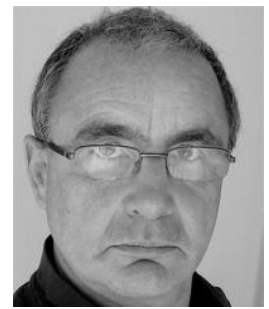

Jacques Ambühl studied Applied Mathematics at EPFL and spent his professional life at the Swiss Federal Office for Meteorology and Climatology. He developed in the eighties of the last century route optimization algorithms for sailing boats engaged in the "Round the World Races". Later, he was a cofounder of the Consortium for Small Scale Modelling (COSMO), aimed at developing and operating local weather forecasting models among European Weather Services. His current field of interest encompasses the interaction between weather forecasting, risk analysis and the development of decision schemes based on bio-inspired artificial intelligence.

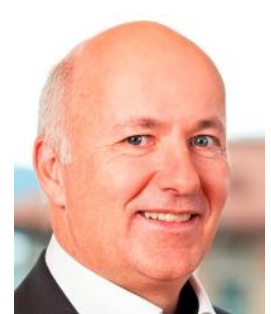

Roland Siegwart is professor for autonomous mobile robots at ETH Zurich. He studied Mechanical Engineering at ETH, brought up a spin-off company, spent ten years as professor at EPFL and he held visiting positions at Stanford University and NASA Ames. He is and was the coordinator of multiple European projects and cofounder of half a dozen spinoff companies. He is recipient of the IEEE RAS Inaba Technical Award, IEEE Fellow and officer of the International Federation of Robotics Research (IFRR). He is in the editorial board of multiple journals in robotics and was a general chair of several conferences in robotics including IROS 2002,
AIM 2007, FSR 2007 and ISRR 2009. His interests are in the design and navigation of wheeled, walking and flying robots operating in complex and highly dynamical environments. 
APPENDIX A

Additional validation case with unsuitable weather conditions
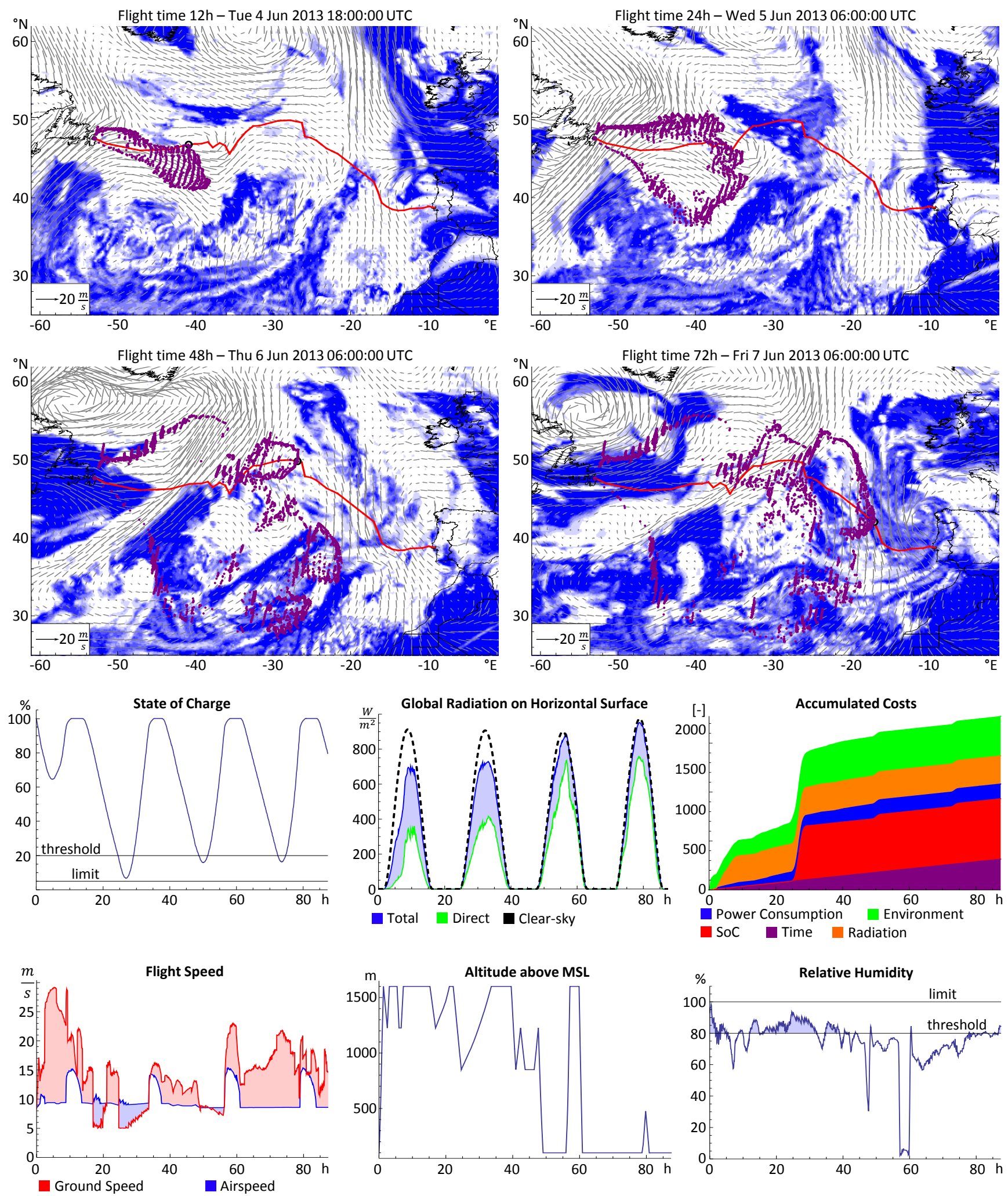

Figure 13 - The validation case with unsuitable weather conditions shows a path that starts with heavy winds and high cloud coverage. In the first night, the SoC is critically low. The airspeed needs to be increased temporarily to maintain a certain ground speed. Costs for $\mathrm{SoC}$, power consumption, radiation and environmental dangers cannot be totally avoided. 